\title{
General Psychiatry Towards a precision psychiatry approach to anxiety disorders with ecological momentary assessment: the example of panic disorder
}

\author{
Donald J Robinaugh (D) , ${ }^{1,2}$ Mackenzie L Brown, ${ }^{1}$ Olivia M Losiewicz, ${ }^{1}$ \\ Payton J Jones, ${ }^{1,3}$ Luana Marques, ${ }^{1,2}$ Amanda W Baker (1D) 1,2
}

To cite: Robinaugh DJ, Brown ML, Losiewicz OM, et al. Towards a precision psychiatry approach to anxiety disorders with ecological momentary assessment: the example of panic disorder. General Psychiatry 2020;33:e100161. doi:10.1136/ gpsych-2019-100161

- Additional material is published online only. To view please visit the journal online (http://dx.doi.org/10.1136/ gpsych-2019-100161).

Received 07 October 2019 Revised 14 November 2019 Accepted 28 November 2019
Check for updates

(c) Author(s) (or their employer(s)) 2020. Re-use permitted under CC BY-NC. No commercial re-use. See rights and permissions. Published by BMJ.

1Department of Psychiatry, Massachusetts General Hospital, Boston, Massachusetts, USA ${ }^{2}$ Department of Psychiatry, Harvard Medical School, Boston, Massachusetts, USA

${ }^{3}$ Department of Psychology, Harvard University, Cambridge, Massachusetts, USA

Correspondence to Dr Donald J Robinaugh; drobinaugh@mgh.harvard.edu

\section{ABSTRACT}

Background Treatments for anxiety disorders are among the most effective in psychiatry. Yet, there is considerable room for improvement.

Aim In this paper, we discuss the value of ecological momentary assessment as a research method and clinical tool.

Methods We begin by describing ecological momentary assessment and its advantages, including the ability to collect ecologically valid information about mental disorders, in real time, in individual patients. We then illustrate the value of this approach for anxiety disorder treatment using two patients with panic disorder who completed ecological momentary assessments for 2 weeks before and after a cognitive-behavioural therapy intervention. We focus especially on two key pieces of information provided by ecological momentary assessment data: information about symptom dynamics and information about the relationships among symptoms as they unfold over time within individual patients.

Perspective Although considerable work is needed to further develop this methodology in the context of anxiety disorder treatment, we believe that these pieces of information may ultimately inform our understanding of how anxiety disorder treatments have their effect and how those treatments can be tailored to individual patients.

During the late 20th century, researchers adopting a cognitive-behavioural perspective developed a series of evidence-based treatments for generalised anxiety disorder, social anxiety disorder and panic disorder. ${ }^{1-6}$ The short-term outcomes of these treatments are among psychiatry's biggest success stories. This is especially true for panic disorder. Just 50 years ago, recurrent panic attacks were viewed as a condition highly resistant to treatment. ${ }^{5}$ Today, treatments for panic disorder are among the most efficacious interventions in psychiatry. However, there is considerable room for improvement. Many patients fail to respond to treatments ${ }^{67}$ and relapse rates are troublingly high. Data from the Netherlands Study of Depression and Anxiety showed that after 2 years, $23.5 \%$ of remitters experienced a recurrence of their anxiety disorder. ${ }^{8}$ Data from the Harvard/Brown Anxiety Disorders Research Program showed that rates of recurrence over a 12-year period are even higher: $56 \%$ for panic disorder without agoraphobia, $39 \%$ for social phobia and $45 \%$ for generalised anxiety disorder. ${ }^{9}$ Perhaps even more troubling, there has been little innovation in the treatment of anxiety disorders in the last 30 years $^{6}$ and there is little clarity about how these treatments can be improved.

In this paper, we suggest that collecting intensive time series data on individual patients using ecological momentary assessment (EMA) can provide a way forward by enriching our understanding of anxiety disorders and their treatments. Further, we argue that this methodology has the potential to guide the development of new interventions and, ultimately, move us towards a 'precision psychiatry' approach in which intensive time series data are used to tailor the treatment of anxiety disorders to individual patients. We begin by providing an overview of EMA and then illustrate two ways in which it can advance our understanding of anxiety disorder treatment: (1) by providing rich, ecologically valid information about the temporal dynamics of individual symptoms and how those dynamics change over treatment, and (2) by equipping researchers to examine the relationships among symptoms as they unfold over time in individual patients. We will illustrate these features using EMA data from two patients who received a cognitivebehavioural treatment for panic disorder.

\section{OVERVIEW OF EMA}

In 1942, Gordon Allport made a call to researchers to use daily life methods in the psychological sciences, stating: "acquaintance with particulars is the beginning of all 
knowledge... psychology needs to concern itself with life as it is lived". ${ }^{10}$ EMA is a methodology that aims to answer this call, emphasising the collection of data in daily life, often using repeated real-time data measurements in subjects' natural environments. ${ }^{11}$ The term 'EMA' was formally introduced in 1994 by Stone and Shiffman ${ }^{12}$ and, in recent decades, EMA has become a widely applied method used to examine social, psychological and physiological processes. ${ }^{11} 13$ The purpose and application of EMA methodology can vary widely from examining the reliability of traditional self-reports to assessing outcomes in treatment trials and causal models, ${ }^{14}$ and there is a growing literature detailing why EMA should be used, how it can be used, and what EMA can tell us above and beyond traditional methods. ${ }^{15-17}$ EMA can also be delivered through a variety of methods (eg, written diaries, smartphone applications and wearable measurement devices) and can include random time sampling (ie, data collected at random time points over the course of a day) or event-based sampling (ie, data recorded when a certain condition is met, such as a subject experiencing a panic attack). However, the core feature of EMA data is that they aim "to capture life as it is lived", ${ }^{13}$ thereby providing detailed ecologically valid data that can be used to examine how social, psychological and physiological processes naturally unfold over time. ${ }^{15}$

EMA research has advantages beyond ecological validity. First, this type of data collection minimises retrospective bias. ${ }^{11} 1518$ Subjects report on their current states, thereby minimising recall biases and focusing on those states with which the subject has the most direct access. ${ }^{16}$ Second, EMA data minimises selectivity when one is describing their experiences by asking a subject to report only on a very specific and very recent timeframe (ie, the last $5 \mathrm{~min}$ ), thus eliminating the likelihood that the subject will have many experiences informing how they respond to the question. ${ }^{16}$ Finally, EMA data provides us with information that is less accessible with traditional methodological approaches, including rich information about processes as they unfold (eg, the rise and fall of anxiety throughout the day), information outside of what subjects would normally attend to (eg, subtle avoidance behaviours) and information that subjects cannot report on their own (eg, psychophysiological arousal on wakening). ${ }^{15}$

\section{EXAMPLE OF PANIC DISORDER}

In the remainder of this paper, we illustrate the value of EMA using the example of two patients who received cognitive-behavioural therapy for panic disorder. We will refer to these patients as Bob and Alice (names have been changed to protect patient confidentiality). Bob was in his 40 s with primary panic disorder and comorbid agoraphobia. He exhibited a pre-treatment Panic Disorder Symptom Severity (PDSS) ${ }^{19}$ score of 21 and a Clinical Global Impression-Severity $\left(\right.$ CGI-S ${ }^{20}$ score of 5, each indicating marked distress and impairment. After treatment, Bob's PDSS score reduced to 5 and his CGI-S score reduced to 2 , indicating he no longer met diagnostic criteria for panic disorder, but some symptoms remained. Alice was in her 20s with primary panic disorder with agoraphobia. She exhibited a pre-treatment PDSS score of 18, also corresponding to markedly ill, and a CGI-S score of 4, signifying more moderate distress and impairment than that reported by Bob. Over the course of treatment, Alice exhibited substantial reductions on the PDSS and CGI-S, scoring a 1 on both measures at post-treatment, indicating full remission of panic disorder.

For a minimum of 2 weeks before and after treatment, Alice and Bob completed EMA assessments five times per day between the hours of 9:00 and 21:00 on their smart phones (see online supplementary materials for more details). At each assessment point, they were asked about 20 different panic symptoms and rated their current experience of that symptom from 0 to 10 . We grouped these symptoms into five categories: cognitive (eg, "I feel like I'm going crazy"), emotional (eg, feeling anxious or panicky), physical (eg, heart racing or feeling dizzy), avoidance behaviour (eg, not going somewhere for fear of having a panic attack) and escape behaviour (eg, leaving somewhere for fear you might have a panic attack). For the analyses presented here, we used the maximum value within each category at each assessment point, as many participants presented with only a specific subset of the symptoms within a given category.

\section{SYMPTOM DYNAMICS}

In the most traditional psychiatric assessments, researchers assess the presence or severity of symptoms over an extended period of time (eg, the past week) and sum those symptom severity reports to provide a 'total score' indexing disorder severity. In this approach, substantial information about the patient is lost. By focusing on 'total scores', we lose information about individual symptoms, often the very things we are intending to target in our treatments. ${ }^{21}$ Moreover, by relying on retrospective recall of a symptom's severity over the past week, we obtain an assessment prone to error and bias, and we typically also limit our understanding to only one aspect of the symptom's dynamics, such as its frequency (eg, the number of panic attacks in the past week) or its mean (eg, the average level of agoraphobic avoidance).

In contrast, EMA provides ecologically valid data that can be used to assess a range of indices regarding a symptom's dynamics. Consider Alice's ratings of panicrelated cognitions before treatment (see figure 1). In a traditional self-report assessment, Alice must reflect on the full range of her panic-related thoughts over these 2 weeks and report the average level of this symptom, a process which is almost certainly highly prone to error. In contrast, in these EMA assessments, Alice needed only to report the extent to which she was having these thoughts in the minutes preceding the survey. Those EMA data can then be used to precisely calculate the mean of panic-related cognitions in the weeks prior to treatment. Perhaps even more importantly, these data allow us to go 
Baseline: Panic Cognitions

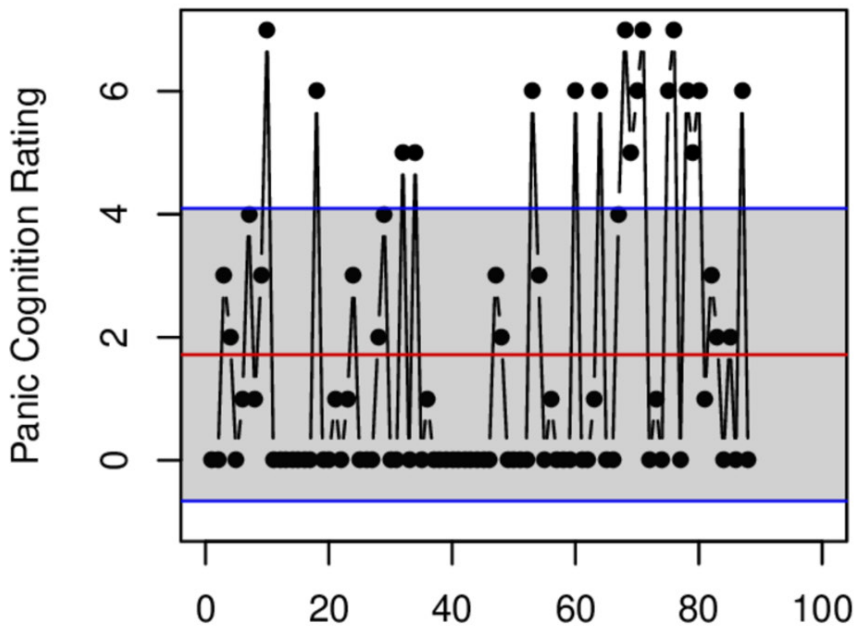

Assessment Point

Endpoint: Panic Cognitions

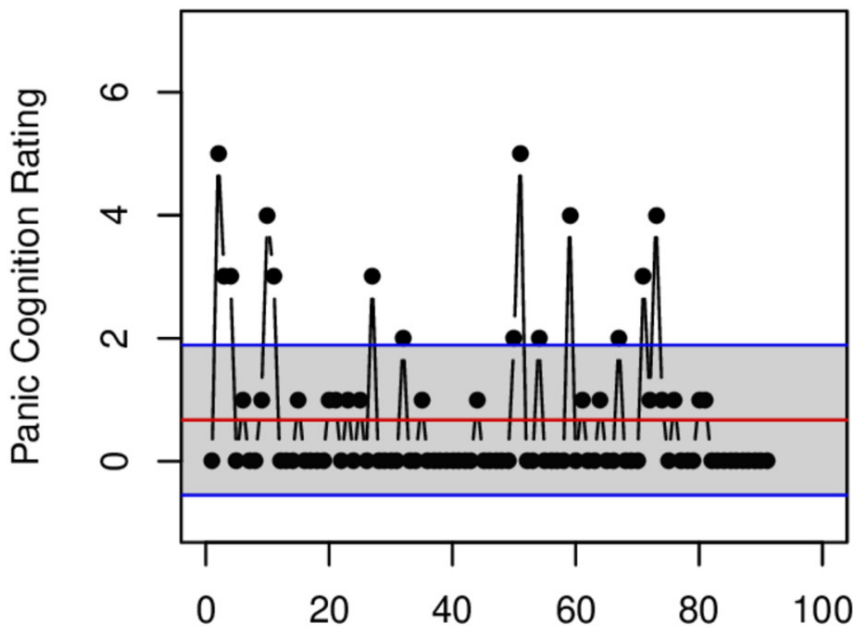

Assessment Point

\section{Endpoint: Anxiety/Panic}

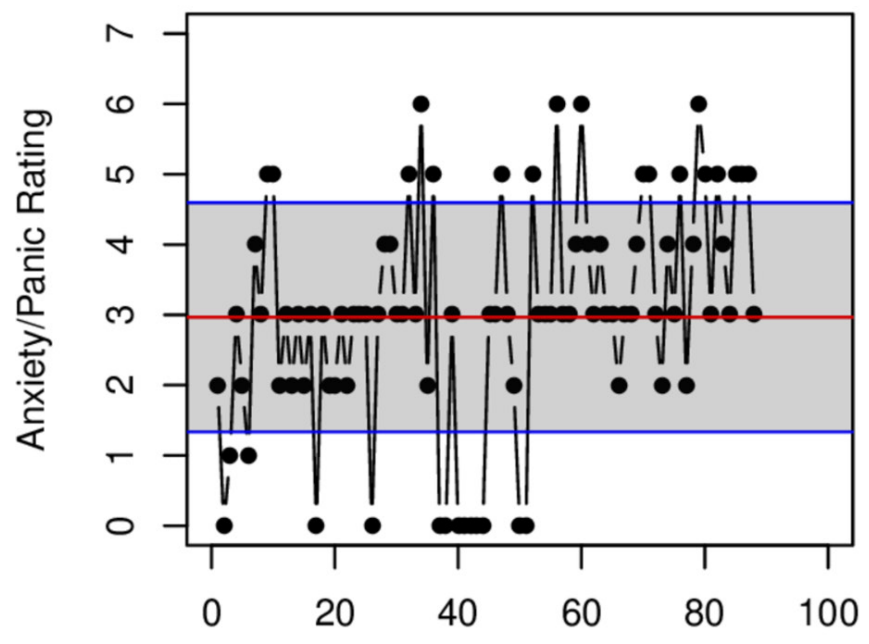

Assessment Point

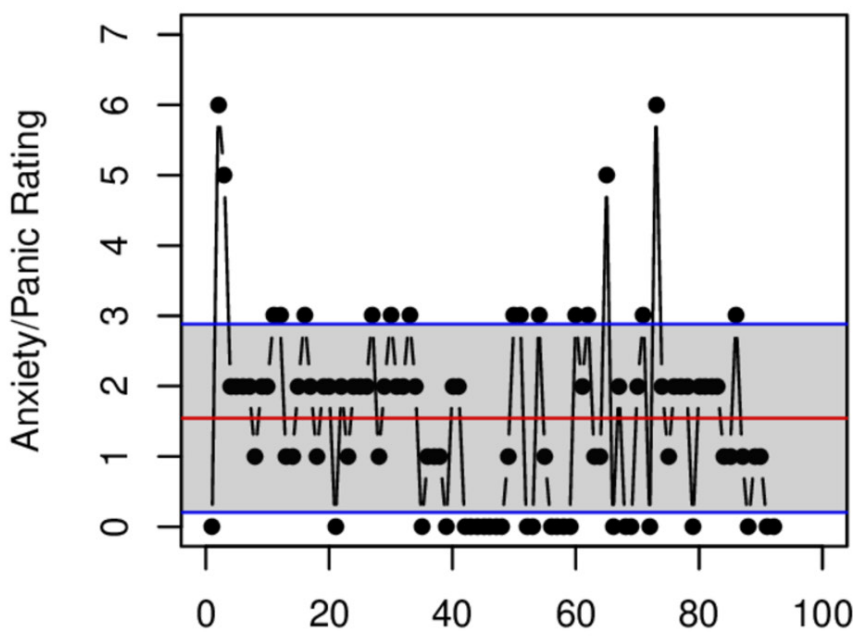

Assessment Point

Figure 1 Panic disorder symptom dynamics pre-treatment and post-treatment. This figure depicts Alice's reports for panicrelated cognitions and feelings of anxiety/panic at each assessment point before and after treatment. The red line represents the mean rating for the symptom over the time period. The blue lines represent the SD. Note: missing values were omitted from this figure.

beyond looking at means alone. Indeed, the mean level of panic-related cognitions $(\mathrm{M}=1.72)$ is a poor summary of Alice's experience prior to treatment, as she rarely reported that particular severity of cognition. Instead, she most commonly reported an absence of panic-related cognitions, punctuated by infrequent but intense experiences with such thoughts.

The symptom dynamics visualised in figure 1 can be quantified in several ways (see table 1). Researchers can calculate the within-person variance of the symptom over a given period of time, providing a richer understanding of the symptom than is provided by the mean alone. For example, Bob reports a comparable mean level of feelings of anxiety and panic as does Alice $(\mathrm{M}(\mathrm{SD})=3.59$ (2.39) and 2.97 (1.63), respectively). However, Bob reports a variance (5.72) nearly twice that of Alice (2.65), immediately identifying Bob as having a very different experience with feelings of anxiety and panic than does Alice; a difference which may warrant a distinct approach in treatment. 
Table 1 Panic disorder symptom dynamics from pretreatment to post-treatment

\begin{tabular}{|c|c|c|c|c|}
\hline & \multicolumn{2}{|l|}{ Alice } & \multicolumn{2}{|l|}{ Bob } \\
\hline & $\begin{array}{l}\text { Pre- } \\
\text { treatment }\end{array}$ & $\begin{array}{l}\text { Post- } \\
\text { treatment }\end{array}$ & $\begin{array}{l}\text { Pre- } \\
\text { treatment }\end{array}$ & $\begin{array}{l}\text { Post- } \\
\text { treatment }\end{array}$ \\
\hline \multicolumn{5}{|l|}{$\begin{array}{l}\text { Avoidance } \\
\text { behaviour }\end{array}$} \\
\hline Mean & 0.44 & 0.02 & 2.53 & 0.56 \\
\hline Variance & 2.16 & 0.02 & 11.59 & 4.04 \\
\hline MSSD & 3.06 & 0.05 & 14.21 & 5.00 \\
\hline Autocorrelation & 0.27 & -0.03 & 0.37 & 0.29 \\
\hline \multicolumn{5}{|l|}{ Escape behaviour } \\
\hline Mean & 0.19 & 0.14 & 2.12 & 1.85 \\
\hline Variance & 0.80 & 0.45 & 12.67 & 8.68 \\
\hline MSSD & 0.96 & 0.64 & 24.44 & 19.37 \\
\hline Autocorrelation & 0.36 & 0.07 & 0.05 & -0.07 \\
\hline \multicolumn{5}{|l|}{$\begin{array}{l}\text { Panic-related } \\
\text { cognitions }\end{array}$} \\
\hline Mean & 1.72 & 0.67 & 4.71 & 4.02 \\
\hline Variance & 5.65 & 1.49 & 7.45 & 3.05 \\
\hline MSSD & 9.69 & 2.21 & 9.05 & 5.59 \\
\hline Autocorrelation & 0.17 & 0.22 & 0.38 & 0.08 \\
\hline \multicolumn{5}{|l|}{$\begin{array}{l}\text { Feelings of } \\
\text { anxiety/panic }\end{array}$} \\
\hline Mean & 2.97 & 1.54 & 3.59 & 3.39 \\
\hline Variance & 2.65 & 1.79 & 5.72 & 3.28 \\
\hline MSSD & 3.58 & 2.81 & 6.63 & 5.98 \\
\hline Autocorrelation & 0.35 & 0.17 & 0.45 & 0.09 \\
\hline \multicolumn{5}{|l|}{$\begin{array}{l}\text { Physical } \\
\text { symptoms }\end{array}$} \\
\hline Mean & 2.16 & 1.12 & 4.12 & 4.37 \\
\hline Variance & 3.35 & 1.38 & 4.83 & 3.13 \\
\hline MSSD & 5.73 & 2.07 & 4.75 & 5.45 \\
\hline Autocorrelation & 0.17 & 0.20 & 0.54 & 0.18 \\
\hline
\end{tabular}

MSSD, mean square of successive differences.

One can also calculate the mean square of successive differences (MSSD) in order to capture the tendency to change between successive assessment points. ${ }^{22}$ Whereas high variability could arise for a variety of reasons (eg, 1 week of relatively low scores, and a second with significantly elevated scores), MSSD more precisely captures instability from one time point to the next, with high MSSD indicating greater moment-to-moment change and lower MSSD indicating greater stability. Similarly, a symptom's autocorrelation (ie, its correlation with itself over time) is an index of a symptom's temporal dependency. ${ }^{23}$ As seen in table 1 , Bob is quite distinct from Alice in the mean of most symptom scores and also in MSSD, especially for avoidance and escape behaviour. It is only for panicrelated cognitions and physical sensations that Alice exhibited comparable instability from one time point to the next. Together, these indices at pre-treatment identify Bob as being more distinct from Alice than would be observed by examining traditional psychiatric assessments alone. Whereas Bob and Alice exhibited comparable scores on the PDSS that identified them as both being markedly ill, Bob exhibited significantly greater mean score, variance and instability for most symptoms in his EMA assessments, suggesting greater severity.

For both Alice and Bob, there were substantial reductions in within-person variance, MSSD and autocorrelation across symptoms from pre-treatment to post-treatment, suggesting a reduced amplitude of these experiences and greater stability over time (notably, reductions in MSSD did not coincide with increases in autocorrelation as one might suspect, likely due to a restricted range of symptoms at post-treatment). For example, from examining Alice's cognitive symptoms from pre-treatment to posttreatment as depicted in figure 1, it is clear that it is not merely the mean of symptoms changing. Peaks of strong panic-related thoughts are less frequent and less severe, suggesting stably ameliorated symptoms by the end of treatment. Similarly, the observed reduction in Alice's mean feelings of anxiety and panic is augmented by the observation that variability and MSSD have reduced as well.

\section{PATIENT-SPECIFIC RELATIONS AMONG SYMPTOMS}

Another advantage of EMA data is that it can inform our understanding of how symptoms relate to one another over time. Historically, psychiatric research has viewed symptoms principally as indicators of an underlying disorder. ${ }^{24}{ }^{25}$ However, researchers have recently argued that causal relations among the symptoms themselves may play an important role in the tendency for these symptoms to cohere as syndromes. ${ }^{26}$ For example, there are clear plausible causal relations among recurrent panic attacks, persistent worry or concern about those attacks, and avoidance of situations in which panic attacks may occur that may lead them to cohere as the syndrome we refer to as panic disorder. Indeed, cognitive-behavioural theories of panic disorder posit that causal relations among these symptoms contribute to their joint persistence over time (eg, avoidance maintains vulnerability to recurrent panic attacks). ${ }^{27}$

From this perspective, mental disorders can be understood and studied as networks of mutually reinforcing symptoms, thereby shifting the focus of psychiatric research from identifying an underlying disorder to instead mapping the relationships among symptoms that contributes to its persistence over time. ${ }^{26} 2829$ Although cross-sectional analyses can inform our understanding of such relationships, analyses based on EMA data have significant advantages in this regard. EMA data allow researchers to assess the relationships among symptoms where and when they occur: within the individual and in real time. ${ }^{30}$ This is critical because findings from crosssectional analyses cannot be assumed to correspond to findings at the level of the individual patient except under very specific conditions that are rarely met in psychiatric 

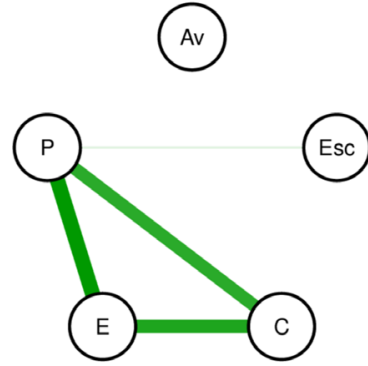

Pre-treatment

\section{Bob}

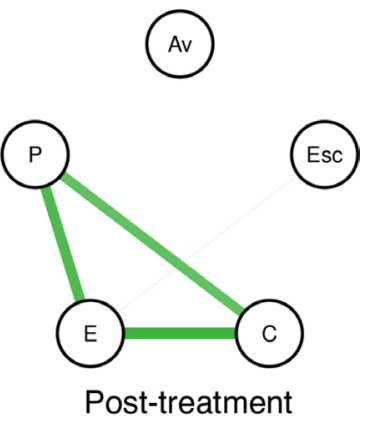

Alice

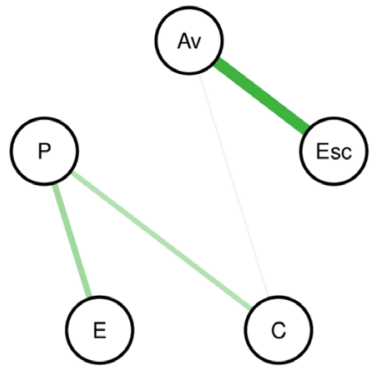

Pre-treatment

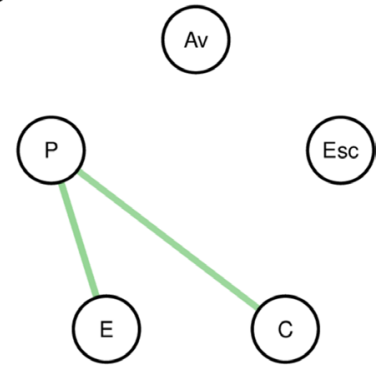

Post-treatment
Figure 2 Intraindividual symptom networks pre-treatment and post-treatment. This figure depicts the relationships among individual symptoms of panic disorder for Bob (top row) and Alice (bottom row) at pre-treatment (left column) and post-treatment (right column). These networks were calculated using the $\mathrm{R}$ package graphicalVAR. Edges represent contemporaneous regularised partial correlations among symptoms. Thicker edges (ie, the line between two symptom 'nodes') indicate a stronger conditional association between those symptoms. A green edge indicates a positive association. Edges are weighted across all four networks such that the maximum edge value among the four graphs is depicted as the thickest line. Av = Avoidance behavior; Esc = Escape behavior; $\mathrm{C}=$ Panic-related cognitions; $\mathrm{E}=$ Panic-related emotions (i.e., anxiety or panic); $\mathrm{P}=$ Physical symptoms

research. ${ }^{1731}$ If you want to understand the relationships among symptoms as they unfold in individual patients, you must assess those relationships using intensive time-series data. Assessing these associations also allows researchers to examine individual differences in how symptoms relate to one another over time, thereby providing new information about how an anxiety disorder operates within an individual patient.

To illustrate this approach, we used a vector autoregressive modelling approach to examine the contemporaneous network of associations among symptoms for Alice and Bob at baseline and at post-treatment. ${ }^{32}$ As seen in figure 2, the structure of relationships among symptoms for Alice and Bob exhibit both similarities and differences. In both patients, physical sensations, panic-related cognitions and feelings of anxiety or panic are strongly related to one another. This finding, at least superficially, appears to align with what may be expected from the cognitive-behavioural theories of panic disorder, which posit causal relations among these symptoms. ${ }^{33}$ For Alice, there is also a strong association between escape and avoidance behaviour, a relationship that is not present in Bob's network. Interestingly, there is reasonable consistency between the networks from pre-treatment to posttreatment for both patients, with the exception that Alice's network no longer features a prominent association between escape and avoidance behaviour, attributable to the fact that she engaged in minimal escape or avoidance at post-treatment.

\section{LIMITATIONS AND CONSIDERATIONS FOR MOVING FORWARD}

Although there are numerous advantages to using an EMA methodological approach, there are, of course, also limitations. Perhaps most saliently, it is more time consuming than a single administration of a self-report questionnaire and more reliant on patients' adherence. It is unclear whether the typical patient will be willing to complete the type of intensive EMA data collection that we have reported here. EMA research also introduces a host of new measurement issues that are not encountered in traditional psychiatric assessments (for an extended discussion, see Schwarz ${ }^{16}$ ). For example, there is evidence suggesting that when respondents are repeatedly asked the same or similar questions by the same researcher, they will attempt to give different answers to avoid providing the same information multiple times and will also attempt to make use of the full breadth of the rating scale, ${ }^{16}$ thereby adding influences on the data beyond the thing being studied. Similarly, the findings from EMA research will strongly depend on the time frames being assessed, particularly the interval between assessments, suggesting that careful attention must be paid when designing an EMA survey protocol.

In addition, we believe it is important to note that, as an emerging area of research, the methods for collecting EMA data and the analytic tools that make use of these data are very much under development. For example, best practice standards for analysing and interpreting mental disorder symptom networks estimated from time-series data are not yet available. More fundamentally, it remains unclear whether symptom dynamics and intraindividual network analyses can indeed lead to better case conceptualisations or improved treatment. Accordingly, our promotion of these analyses here should not be regarded as a call to immediately implement these procedures into daily practice, but rather to highlight their potential and call for further research investigating these methods and their potential clinical utility. For further reading on how to develop and carry out research using EMA methods, please see, ${ }^{111516}$ and for more information on intraindividual network analyses, please see. ${ }^{32} 34$

\section{PERSPECTIVES}

As we have reviewed here, EMA provides data that are rich and ecologically valid. Moreover, these data can provide 
new information about symptom dynamics and the relationships among symptoms as they unfold over time in individual patients; information that would be difficult or impossible to assess with traditional psychiatric assessments. Accordingly, we believe that EMA research has tremendous promise to inform the future of anxiety disorder treatment research. We believe it can do so in two critical ways. First, the data on symptom dynamics and intraindividual associations among symptoms have the potential to substantially inform our understanding of how treatments have their effect. We are especially interested in the use of these methods to investigate temporal sequencing of symptom reductions (eg, reductions in avoidance that precede reductions in feelings of anxiety or panic) and to clarify the precise aspects of psychiatric disorders most affected by treatment (eg, reducing the instability rather than the mean of panic-related physical sensations). Second, the rich information provided by EMA data paves the way towards better understanding our patients, providing information not only about their specific constellation of symptoms, but also the dynamics of those symptoms and the relationships among them. This information will allow us to investigate whether all patients who benefit from treatment benefit in the same way or if different patients experience gains in different symptoms; information that could ultimately inform a personalised treatment approach. ${ }^{35}$ Accordingly, it will be critical for researchers to continue determining the best way to collect, analyse and interpret data using this highly promising method.

Contributors All authors contributed to the initial conceptualisation of this article. OML conducted all statistical analyses under the supervision of DJR. MLB and OML produced the initial draft of the article. DJR, MLB, OML, PJ and AWB all contributed to the subsequent development of the manuscript.

Funding This study was supported by a National Institute of Mental Health Career Development Award (1K23MH113805-01A1), a Brain and Behavior Research Foundation NARSAD Award, and a Harvard Catalyst/The Harvard Clinical and Translational Science Center (National Center for Research Resources and the National Center for Advancing Translational Sciences), National Institutes of Health Award (KL2 TR001100).

Competing interests None declared.

Patient consent for publication Not required.

Ethics approval We confirm that the conduct of human subjects mentioned in the manuscript was conducted with the ethical approval of all relevant bodies. Institutional Review Board number: 00003136; study protocol ID: 2011 P001365.

Provenance and peer review Commissioned; externally peer reviewed.

Data availability statement Data are available upon request

Open access This is an open access article distributed in accordance with the Creative Commons Attribution Non Commercial (CC BY-NC 4.0) license, which permits others to distribute, remix, adapt, build upon this work noncommercially, and license their derivative works on different terms, provided the original work is properly cited, appropriate credit is given, any changes made indicated, and the use is non-commercial. See: http://creativecommons.org/ licenses/by-nc/4.0/.

\section{ORCID iDs}

Donald J Robinaugh http://orcid.org/0000-0001-5560-7035

Amanda W Baker http://orcid.org/0000-0002-0103-5411
REFERENCES

1 Sánchez-Meca J, Rosa-Alcázar Al, Marín-Martínez F, et al. Psychological treatment of panic disorder with or without agoraphobia: a meta-analysis $\{$. Clin Psychol Rev 2010;30:37-50.

2 Cuijpers P, Sijbrandij M, Koole S, et al. Psychological treatment of generalized anxiety disorder: a meta-analysis. Clin Psychol Rev . 2014;34:130-40.

3 Cuijpers P, Gentili C, Banos RM, et al. Relative effects of cognitive and behavioral therapies on generalized anxiety disorder, social anxiety disorder and panic disorder: a meta-analysis. J Anxiety Disord 2016;43:79-89.

4 Carpenter JK, Andrews LA, Witcraft SM, et al. Cognitive behavioral therapy for anxiety and related disorders: a metaanalysis of randomized placebo-controlled trials. Depress Anxiety 2018;35:502-14.

5 McNally RJ. Panic disorder: a critical analysis. New York, NY: Guilford Press, 1994.

6 Schmidt NB, Keough ME. Treatment of panic. Annu Rev Clin Psychol 2010;6:241-56.

7 Roy-Byrne P. Treatment-refractory anxiety; definition, risk factors, and treatment challenges. Dialogues Clin Neurosci 2015;17:191-206.

8 Scholten WD, Batelaan NM, van Balkom AJLM, et al. Recurrence of anxiety disorders and its predictors. J Affect Disord 2013;147:180-5.

9 Bruce SE, Yonkers KA, Otto MW, et al. Influence of psychiatric comorbidity on recovery and recurrence in generalized anxiety disorder, social phobia, and panic disorder: a 12-year prospective study. AJP 2005;162:1179-87.

10 Allport G. The use of personal documents in psychological science. Soc Sci Res Counc Bull 49, 1942. Available: https://archive.org/ details/useofpersonaldoc00allprich [Accessed 11 Nov 2019].

11 Shiffman S, Stone AA, Hufford MR. Ecological momentary assessment. Annu Rev Clin Psychol 2008;4:1-32.

12 Stone AA, Shiffman S. Ecological momentary assessment (EMA). Behav Med Ann Behav Med 1994;16:199-202.

13 Bolger N, Davis A, Rafaeli E. Diary methods: capturing life as it is lived. Annu Rev Psychol 2003;54:579-616.

14 Cain AE, Depp CA, Jeste DV. Ecological momentary assessment in aging research: a critical review. J Psychiatr Res 2009;43:987-96.

15 Reis HT. Why researchers should think "real-world": a conceptual rationale. In: Mehl MR, Conner TS, eds. Handbook of research methods for studying daily life. New York, NY: The Guilford Press, 2012: 3-17.

16 Schwarz N. Why researchers should think "real-time": a cognitive rationale. In: Mehl MR, Conner TS, eds. Handbook of research methods for studying daily life. New York, NY: The Guilford Press, 2012: 22-42.

17 Hamaker EL. Why researchers should think "within-person": a paradigmatic rationale. In: Mehl MR, Conner TS, eds. Handbook of research methods for studying daily life. New York, NY: The Guilford Press, 2012: 43-61.

18 Bos FM, Schoevers RA, aan het Rot M. Experience sampling and ecological momentary assessment studies in psychopharmacology: a systematic review. Eur Neuropsychopharmacol 2015;25:1853-64.

19 Shear MK, Brown TA, Barlow DH, et al. Multicenter collaborative panic disorder severity scale. AJP 1997;154:1571-5.

20 Guy W. ECDEU assessment manual for psychopharmacology: publication ADM 76-338. Washington, DC: US Department of Health, Education and Welfare, 1976: 217-22.

21 Fried El, Nesse RM. Depression sum-scores don't add up: why analyzing specific depression symptoms is essential. BMC Med 2015;13:1-11.

22 Jahng S, Wood PK, Trull TJ. Analysis of affective instability in ecological momentary assessment: indices using successive difference and group comparison via multilevel modeling. Psychol Methods 2008;13:354-75.

23 Koval P, Ogrinz B, Kuppens P, et al. Affective instability in daily life is predicted by resting heart rate variability. PLoS One 2013;8:e81536.

24 Kendler KS. From many to one to many - the search for causes of psychiatric illness. JAMA Psychiatry 2019;76.

25 Radden J. Rethinking disease in psychiatry: disease models and the medical imaginary. J Eval Clin Pract 2018;24:1087-92.

26 Borsboom D, Cramer AOJ. Network analysis: an integrative approach to the structure of psychopathology. Annu Rev Clin Psychol 2013;9:91-121.

27 Goldstein AJ, Chambless DL. A reanalysis of agoraphobia. Behav Ther 1978;9:47-59.

28 Cramer AOJ, Waldorp LJ, van der Maas HLJ, et al. Comorbidity: a network perspective. Behav Brain Sci 2010;33:137-50.

29 Schmittmann VD, Cramer AOJ, Waldorp LJ, et al. Deconstructing the construct: a network perspective on psychological phenomena. New Ideas Psychol . 2013;31:43-53. 
30 Hamaker EL, Wichers M. No time like the present: discovering the hidden dynamics in intensive longitudinal data. Curr Dir Psychol Sci 2017;26:10-15

31 Molenaar PCM, Campbell CG. The new person-specific paradigm in psychology. Curr Dir Psychol Sci 2009;18:112-7.

32 Epskamp S, van Borkulo CD, van der Veen DC, et al. Personalized network modeling in psychopathology: the importance of contemporaneous and temporal connections. Clin Psychol Sci 2018;6:416-27.
33 Clark DM. A cognitive approach to panic. Behav Res Ther 1986;24:461-70.

34 Bringmann LF, Pe ML, Vissers N, et al. Assessing temporal emotion dynamics using networks. Assessment 2016;23:425-35.

35 Mofsen AM, Rodebaugh TL, Nicol GE, et al. When all else fails, listen to the patient: a viewpoint on the use of ecological momentary assessment in clinical trials. JMIR Ment Health 2019;6.

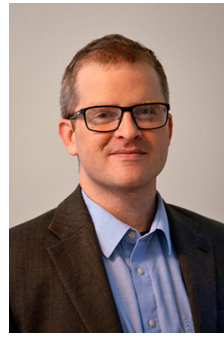

Donald J. Robinaugh received his PhD from Harvard University in 2015 under the mentorship of Dr. Richard McNally. Since 2018, he has worked as a clinical psychologist at the Center for Anxiety and Traumatic Stress Disorders and Complicated Grief Program in the Department of Psychiatry at Massachusetts General Hospital and as an Assistant Professor of Psychiatry at Harvard Medical School. His research interest primarily focuses on using computational psychiatry and the tools of network science to investigate mental disorders as complex systems. He is especially focused on applying these tools in the context of anxiety disorders and complicated grief. 